\title{
EXTREMES OF INTERARRIVAL TIMES OF A POISSON PROCESS UNDER CONDITIONING
}

1. Introduction. Consider a homogeneous Poisson process $\{N(t)$ : $t \geq 0\}$ with parameter $\lambda=E(N(1)), \lambda>0$. Let $0=S_{0}<S_{1}<\ldots$ denote the successive times of events of the process and for $j \geq 1, Y_{j}=S_{j}-S_{j-1}$ be the interarrival times.

It is known that under $N(t)=n, t>0$, the random variables $0 \leq$ $S_{1} \leq \ldots \leq S_{n} \leq t$ are distributed as the order statistics of a sample of $n$ observations taken from the uniform distribution on $[0, t]$. This represents the most natural relationship between the Poisson process, random points on a line, and the uniform distribution, random points on an interval. For example, see Pyke [6].

Let $0=X_{0, n}<X_{1, n}<\ldots<X_{n, n}<t=X_{n+1, n}$ be the order statistics corresponding to a sample from the uniform distribution on $[0, t], t>0$. If for $1 \leq j \leq n+1$, we let $Y_{j}=X_{j, n}-X_{j-1, n}$ be the spacings, then it is known that (for example, see [1]) as $n \rightarrow \infty$,

$$
P\left\{\max _{1 \leq j \leq n+1} Y_{j} \leq\left(\frac{\log n+x}{n}\right) t\right\} \rightarrow \exp (-\exp (-x)) .
$$

Thus, in the case when $Y_{j}$ are the interarrival times of a homogeneous Poisson process, the above implies that as $n \rightarrow \infty$,

$$
P\left\{\max _{1 \leq j \leq n+1} Y_{j} \leq\left(\frac{\log n+x}{n}\right) t \mid N(t)=n\right\} \rightarrow \exp (-\exp (-x)) .
$$

This can also be found in [5].

The purpose of this paper is to investigate the limiting distribution of the extremes of the interarrival times, $Y_{j}$, of a Poisson process under a variety of conditioning. In Section 2, we discuss the main results and in Section 3 we consider the limiting distribution of the $k$ th extremes. Finally, in Section 4

1991 Mathematics Subject Classification: Primary 60K05.

Key words and phrases: Poisson process, extreme, exchangeable. 
we consider the asymptotic distribution for the number of interarrival times satisfying certain inequalities.

2. Main result. The main results are the following theorems.

Theorem 1. Let $Y_{j}$ be the interarrival times of a homogeneous Poisson process. If $m=m(n), n=1,2, \ldots$, is a sequence of positive integers such that $m \rightarrow \infty$ and $m / n \rightarrow 0$ as $n \rightarrow \infty$, then for any real number $x$,

$$
\lim _{n \rightarrow \infty} P\left\{\max _{1 \leq j \leq m} Y_{j} \leq\left(\frac{\log m+x}{n}\right) t \mid N(t)=n\right\}=\exp (-\exp (-x)) .
$$

In what follows, we let $G(x)=\exp (-\exp (-x)), Z_{m}=\max _{1 \leq j \leq m} Y_{j}$ and $T_{n}(x)=(\log m+x) / n$, where $m=m(n)$ is a sequence of positive integers such that $m \rightarrow \infty$ and $m / n \rightarrow 0$ as $n \rightarrow \infty$.

The result in Theorem 1 can also be extended to the cases where we condition on $N(t) \geq n$ rather than $N(t)=n$ and let $t=t(n)$ instead of being fixed. That is, we will also prove that the following theorem holds.

TheOREM 2. Let $Y_{j}$ be the interarrival times of a homogeneous Poisson process. If $m=m(n), n=1,2, \ldots$, is a sequence of positive integers such that $m \rightarrow \infty$ and $m / n \rightarrow 0$ as $n \rightarrow \infty$, then for any real number $x$,

$$
\begin{gathered}
\lim _{n \rightarrow \infty} P\left\{Z_{m} \leq T_{n}(x) t \mid N(t) \geq n\right\}=G(x), \\
\lim _{n \rightarrow \infty} P\left\{Z_{m} \leq T_{n}(x) t(n) \mid N(t(n))=n\right\}=G(x),
\end{gathered}
$$

where $t(n)>0$ for all $n$, and

$$
\lim _{n \rightarrow \infty} P\left\{Z_{m} \leq T_{n}(x) t(n) \mid N(t(n)) \geq n\right\}=G(x),
$$

where $t(n)>0$ and $t(n)=o(n)$ as $n \rightarrow \infty$.

Rem ark. It is easy to see that under $N(t)=n$, the random variables $Z_{j}^{(n)}=(n / t) Y_{j}$ are identically distributed with distribution $F_{n}(x)=1-$ $(1-x / n)^{n}$. Moreover, we see that under $N(t)=n, Z_{j}^{(n)}, 1 \leq j \leq m$, are exchangeable but not independent. However, observe that $F_{n}(x) \rightarrow 1-e^{-x}$ as $n \rightarrow \infty$ and so $Z_{j}^{(n)}$ are asymptotically exponentially distributed. On the other hand, if $X_{j}$ are independent and identically distributed exponential distributions, then

$$
P\left\{\max _{1 \leq j \leq n} X_{j} \leq \log n+x\right\}=\left(1-\frac{e^{-x}}{n}\right)^{n} \rightarrow G(x)
$$

as $n \rightarrow \infty$. Hence Theorems 1 and 2 can be viewed as extensions of the above result.

In the proofs of Theorems 1 and 2 we will use the following result. 
LEMMA 1. If $m=m(n), n=1,2, \ldots$, is a sequence of positive integers such that $m \rightarrow \infty$ and $m / n \rightarrow 0$ as $n \rightarrow \infty$, then for any real number $x$, and fixed $k=1,2,3, \ldots$,

$$
\lim _{n \rightarrow \infty} m^{k}\left(1-k T_{n}(x)\right)^{n}=e^{-k x} .
$$

Proof. It suffices to show that

$$
k \log m+n \log \left(1-k T_{n}(x)\right) \rightarrow-k x \quad \text { as } n \rightarrow \infty .
$$

To see this, we first observe that for $x>0$,

$$
\log (1-x)=-x-\frac{1}{2} \frac{x^{2}}{(1-\zeta(x))^{2}} \quad \text { for } 0 \leq \zeta(x) \leq x .
$$

Thus, it follows that for large $n$,

$$
\log \left(1-k T_{n}(x)\right)=-k T_{n}(x)-\frac{1}{2} \frac{k^{2}}{\left(1-\zeta_{n}(x)\right)^{2}} T_{n}^{2}(x),
$$

where $0 \leq \zeta_{n}(x) \leq k T_{n}(x)$. Hence, we see that

$$
k \log m+n \log \left(1-k T_{n}(x)\right)=-k x-\frac{k^{2}}{2\left(1-\zeta_{n}(x)\right)^{2}} n T_{n}^{2}(x) .
$$

Since $\lim _{n \rightarrow \infty} \zeta_{n}(x)=0$ and $\lim _{n \rightarrow \infty} n T_{n}^{2}(x)=\lim _{n \rightarrow \infty}(\log m+x)^{2} / n=0$, we see that the above implies the desired result.

The following lemma is an easy consequence of the fact that under $N(t)=n, Y_{1}, \ldots, Y_{n}$ are distributed as the spacings of the order statistic of a sample of $n$ observations from the uniform distribution on $[0, t]$.

Lemma 2. Under $N(t)=n$, the interarrival times $Y_{1}, \ldots, Y_{n}$ are exchangeable random variables and for $x>0$,

$$
P\left\{Y_{1}>x, Y_{2}>x, \ldots, Y_{k}>x \mid N(t)=n\right\}=\left(1-\frac{k x}{t}\right)_{+}^{n}
$$

for $k=1, \ldots, n$, where $a_{+}=\max \{a, 0\}$.

The proofs of Theorems 1 and 2 are based on the following theorem due to Ridler-Rowe [7] and D. J. Kendall [4]. The proof can also be found in Galambos [2], p. 309.

TheOREM 3. Consider a sequence of probability spaces and let $A_{1}^{(n)}, \ldots$, $A_{n}^{(n)}$ be exchangeable events on the $n$-th space. Assume $m=m(n) \leq n$ is a sequence of integers such that $m(n) \rightarrow \infty$ with $n$ and that, for some $0<a<\infty$,

$$
\begin{aligned}
m(n) P_{n}\left(A_{1}^{(n)}\right) & \rightarrow a, \\
m^{2}(n) P_{n}\left(A_{1}^{(n)} \cap A_{2}^{(n)}\right) & \rightarrow a^{2} \quad \text { as } n \rightarrow \infty .
\end{aligned}
$$


If $N_{m}=N_{m}^{(n)}$ denotes the number of $A_{j}^{(n)}, 1 \leq j \leq m$, that occur and if $m / n \rightarrow 0$ as $n \rightarrow \infty$, then

$$
\lim _{n \rightarrow \infty} P_{n}\left\{N_{m}=s\right\}=\frac{a^{s} e^{-a}}{s !}, \quad s=0,1,2, \ldots
$$

Proof of Theorem 1 . For $j=1, \ldots, n$ define the events $A_{j}^{(n)}$ by

$$
A_{j}^{(n)}=\left\{Y_{j}>T_{n}(x) t\right\} .
$$

Under the condition that $N(t)=n$, we see that the events $A_{j}^{(n)}, 1 \leq j \leq n$, are exchangeable. Furthermore,

$$
P_{n}\left(A_{1}^{(n)}\right)=P\left\{Y_{1}>T_{n}(x) t \mid N(t)=n\right\}
$$

and

$$
P_{n}\left(A_{1}^{(n)} \cap A_{2}^{(n)}\right)=P\left\{Y_{1}>T_{n}(x) t, Y_{2}>T_{n}(x) t \mid N(t)=n\right\} .
$$

Thus, by Lemma 2 , it follows that

$$
m P_{n}\left(A_{1}^{(n)}\right)=m\left(1-T_{n}(x)\right)^{n}
$$

and

$$
m^{2} P_{n}\left(A_{1}^{(n)} \cap A_{2}^{(n)}\right)=m^{2}\left(1-2 T_{n}(x)\right)^{n} .
$$

Therefore, from Lemma 1 we conclude that

$$
\lim _{n \rightarrow \infty} m P_{n}\left(A_{1}^{(n)}\right)=e^{-x}
$$

and

$$
\lim _{n \rightarrow \infty} m^{2} P_{n}\left(A_{1}^{(n)} \cap A_{2}^{(n)}\right)=e^{-2 x}
$$

If we now let $N_{m}=N_{m}^{(n)}=$ the number of $A_{j}^{(n)}, 1 \leq j \leq m$, that occur, then Theorem 3 implies that

$$
\lim _{n \rightarrow \infty} P_{n}\left\{N_{m}=0\right\}=\exp (-\exp (-x))=G(x)
$$

On the other hand, since

$$
P_{n}\left\{N_{m}=0\right\}=P\left\{Z_{m} \leq T_{n}(x) t \mid N(t)=n\right\}
$$

we see that the theorem holds.

Proof of Theorem 2. (i) If we let

$$
G_{k}(x, t)=P\left\{Z_{m} \leq T_{n}(x) t \mid N(t)=k\right\}
$$

for $k=n, n+1, \ldots$, then we observe that

$$
P\left\{Z_{m} \leq T_{n}(x) t \mid N(t) \geq n\right\}=\frac{1}{P\{N(t) \geq n\}} \sum_{k=n}^{\infty} G_{k}(x, t) e^{-\lambda t} \frac{(\lambda t)^{k}}{k !} .
$$


Next, we claim that as $n \rightarrow \infty$,

$$
P\{N(t) \geq n\} \sim P\{N(t)=n\}
$$

and

$$
\sum_{k=n}^{\infty} G_{k}(x, t) e^{-\lambda t} \frac{(\lambda t)^{k}}{k !} \sim G_{n}(x, t) e^{-\lambda t} \frac{(\lambda t)^{n}}{n !} .
$$

Then the above will imply that as $n \rightarrow \infty$,

$$
P\left\{Z_{m} \leq T_{n}(x) t \mid N(t) \geq n\right\} \sim G_{n}(x, t) .
$$

Since $G_{n}(x, t) \rightarrow G(x)$ as $n \rightarrow \infty$ by Theorem 1 , we see that it suffices to prove the above claim. First, we note that

$$
P\{N(t) \geq n\}=\sum_{k=n}^{\infty} e^{-\lambda t} \frac{(\lambda t)^{k}}{k !}=e^{-\lambda t} \frac{(\lambda t)^{n}}{n !}\left(1+A_{n}(t)\right),
$$

where

$$
A_{n}(t)=\sum_{k=n+1}^{\infty} \frac{(\lambda t)^{k-n}}{(n+1)(n+2) \ldots(n+(k-n))} .
$$

However, for large $n$,

$$
0 \leq A_{n}(t) \leq \sum_{k=n+1}^{\infty}\left(\frac{\lambda t}{n+1}\right)^{k-n}=\frac{\lambda t}{n+1-\lambda t}
$$

Thus, $\lim _{n \rightarrow \infty} A_{n}(t)=0$ and so we see that our first claim holds. Also,

$$
\sum_{k=n}^{\infty} G_{k}(x, t) e^{-\lambda t} \frac{(\lambda t)^{k}}{k !}=G_{n}(x, t) e^{-\lambda t} \frac{(\lambda t)^{n}}{n !}\left(1+\frac{B_{n}(x, t)}{G_{n}(x, t)}\right)
$$

where

$$
B_{n}(x, t)=\sum_{k=n+1}^{\infty} G_{k}(x, t) \frac{(\lambda t)^{k-n}}{(n+1)(n+2) \ldots(n+(k-n))} .
$$

But, since $0 \leq B_{n}(x, t) \leq A_{n}(t)$ we conclude that $\lim _{n \rightarrow \infty} B_{n}(x, t)=0$. This clearly implies our second claim.

(ii) We omit the proof since it is similar to the proof of Theorem 1 .

(iii) The proof is similar to that of (i). Following the proof of (i) we see that $t(n)=o(n)$ implies that $\lim _{n \rightarrow \infty} A_{n}(t)=0$. Thus, applying (ii) we see that the result holds.

Next, we prove the following.

Corollary 1. Let $m=m(n), n=1,2, \ldots$, be a sequence of positive integers such that $m \rightarrow \infty$ and $m / n \rightarrow 0$ as $n \rightarrow \infty$. If $\left\{a_{n}\right\}$ is a sequence 
of positive numbers such that $a_{n} \log m \rightarrow \infty$ as $n \rightarrow \infty$, then

$$
P\left\{\left|\frac{n}{t \log m} Z_{m}-1\right|>a_{n} \mid N(t)=n\right\} \rightarrow 0 \quad \text { as } n \rightarrow \infty .
$$

Proof. Let

$$
G_{n}(x)=P\left\{Z_{m} \leq T_{n}(x) t \mid N(t)=n\right\} .
$$

Then, by Theorem $1, G_{n}(x) \rightarrow G(x)$ as $n \rightarrow \infty$ and since $G$ is continuous we see that this convergence is uniform in $x$. Thus,

$$
\begin{aligned}
& P\left\{\left|\frac{n}{t \log m} Z_{m}-1\right|>a_{n} \mid N(t)=n\right\} \\
& \quad=1-G_{n}\left(a_{n} \log m\right)+G_{n}\left(-a_{n} \log m\right) \\
& \quad \leq 2 \sup _{x}\left|G_{n}(x)-G(x)\right|+1-G\left(a_{n} \log m\right)+G\left(-a_{n} \log m\right) \rightarrow 0
\end{aligned}
$$

as $n \rightarrow \infty$.

Next, we study the asymptotic properties under $N(t)=n$ of $W_{m}=$ $\min _{1 \leq j \leq m} Y_{j}$ and $Z_{m}$, where $m=m(n)$ such that $m \rightarrow \infty$ and $m / n \rightarrow 0$ as $n \rightarrow \infty$. For $a>0$, we see that

$$
\lim _{n \rightarrow \infty} P\left\{W_{m}>\frac{a t}{m n} \mid N(t)=n\right\}=\lim _{n \rightarrow \infty}\left(1-\frac{a}{n}\right)^{n}=e^{-a}
$$

and from Theorem 1 , we know that for any $x$,

$$
\lim _{n \rightarrow \infty} P\left\{Z_{m} \leq T_{n}(x) t \mid N(t)=n\right\}=G(x) .
$$

We now prove that $W_{m}$ and $Z_{m}$ are asymptotically independent.

THEOREM 4. Let $m=m(n)$ be a sequence of positive integers such that $m \rightarrow \infty$ and $m / n \rightarrow 0$ as $n \rightarrow \infty$. Then $W_{m}$ and $Z_{m}$ are asymptotically independent. That is, for $a, b>0$, we have

$$
\lim _{n \rightarrow \infty} P\left\{W_{m}>\frac{a t}{m n}, Z_{m} \leq T_{n}(-\log b) t \mid N(t)=n\right\}=e^{-(a+b)} .
$$

Proof. For $j=1, \ldots, n$, let

$$
A_{j}^{(n)}=\left\{Y_{j} \leq \frac{a t}{m n}\right\} \cup\left\{Y_{j}>T_{n}(-\log b) t\right\} .
$$

Under $N(t)=n, A_{j}^{(n)}$ are clearly exchangeable events. Furthermore, since

$$
P\left(A_{1}^{(n)} \mid N(t)=n\right)=1-\left(1-\frac{a}{m n}\right)^{n}+\left(1-T_{n}(-\log b)\right)^{n}
$$

it follows that

$$
\lim _{n \rightarrow \infty} m P\left(A_{1}^{(n)} \mid N(t)=n\right)=a+b .
$$


Next, we observe that

$$
P\left(A_{1}^{(n)} \cap A_{2}^{(n)} \mid N(t)=n\right)=B_{n}+2 C_{n}+D_{n},
$$

where

and

$$
\begin{aligned}
B_{n} & =P\left\{Y_{j} \leq \frac{a t}{m n} ; j=1,2 \mid N(t)=n\right\} \\
& =1-2\left(1-\frac{a}{m n}\right)^{n}+\left(1-\frac{2 a}{m n}\right)^{n} \\
C_{n} & =P\left\{Y_{1} \leq \frac{a t}{m n} ; Y_{2}>T_{n}(-\log b) t \mid N(t)=n\right\} \\
& =\left(1-T_{n}(-\log b)\right)^{n}-\left(1-T_{n}(-\log b)-\frac{a}{m n}\right)^{n},
\end{aligned}
$$

$$
D_{n}=P\left\{Y_{j}>T_{n}(-\log b) t ; j=1,2 \mid N(t)=n\right\}=\left(1-2 T_{n}(-\log b)\right)^{n} .
$$

Then we see that as $n \rightarrow \infty, m^{2} B_{n} \rightarrow a^{2}, m^{2} C_{n} \rightarrow a b$ and $m^{2} D_{n} \rightarrow b^{2}$. The result now follows by Theorem 3 .

Remark. It can be shown that Theorem 4 holds by conditioning on $N(t) \geq n$ rather than $N(t)=n$.

3. Asymptotic distribution of the $k$ th extremes. If we let $Y_{1, m} \leq$ $Y_{2, m} \leq \ldots \leq Y_{m, m}$ denote the order statistics of $Y_{1}, \ldots, Y_{m}$, then, for a given fixed value of $k, Y_{k, m}$ and $Y_{m-k+1, m}$ are called the $k$ th lower and the $k$ th upper extremes, respectively. Observe that $Y_{1, m}=W_{m}$ and $Y_{m, m}=Z_{m}$.

For $j=1, \ldots, n$, let $A_{j}^{(n)}$ and $N_{m}^{(n)}$ be as defined in the proof of Theorem 1 . Then the proof of Theorem 1 implies that for $s=0,1,2, \ldots$,

$$
\lim _{n \rightarrow \infty} P\left\{N_{m}=s \mid N(t)=n\right\}=\frac{e^{-x s}}{s !} G(x) .
$$

However, we see that

$$
P\left\{Y_{m-k+1, m} \leq T_{n}(x) t \mid N(t)=n\right\}=\sum_{s=0}^{k-1} P\left\{N_{m}=s \mid N(t)=n\right\} .
$$

Thus, from the above, the following theorem holds.

THEOREM 5. Let $m=m(n), n=1,2, \ldots$, be a sequence of positive integers such that $m \rightarrow \infty$ and $m / n \rightarrow 0$ as $n \rightarrow \infty$. Then for any fixed $k \geq 1$, and $-\infty<x<\infty$, we have

$$
\lim _{n \rightarrow \infty} P\left\{Y_{m-k+1, m} \leq T_{n}(x) t \mid N(t)=n\right\}=\sum_{s=0}^{k-1} G(x) \frac{e^{-x s}}{s !} .
$$


Remark. Note that if we let $k=1$ in the above theorem, then we get Theorem 1.

For the $k$ th lower extreme, we can prove that the following theorem holds.

THEOREM 6. Let $m=m(n)$ be a sequence of positive integers such that $m \rightarrow \infty$ and $m / n \rightarrow 0$ as $n \rightarrow \infty$. Then for any fixed $k \geq 1$ and $a>0$, we have

$$
\lim _{n \rightarrow \infty} P\left\{Y_{k, m}>\frac{a t}{m n} \mid N(t)=n\right\}=\sum_{s=0}^{k-1} e^{-a} \frac{a^{s}}{s !} .
$$

Proof. For $j=1, \ldots, n$, let

$$
A_{j}^{(n)}=\left\{Y_{j} \leq \frac{a t}{m n} \mid N(t)=n\right\} .
$$

Then it is easy to show that

$$
\lim _{n \rightarrow \infty} m P_{n}\left(A_{1}^{(n)}\right)=\lim _{n \rightarrow \infty} m P\left(A_{1}^{(n)} \mid N(t)=n\right)=a
$$

and

$$
\lim _{n \rightarrow \infty} m^{2} P_{n}\left(A_{1}^{(n)} \cap A_{2}^{(n)}\right)=\lim _{n \rightarrow \infty} m^{2} P\left(A_{1}^{(n)} \cap A_{2}^{(n)} \mid N(t)=n\right)=a^{2} .
$$

Since $A_{j}^{(n)}$ are exchangeable events, we conclude from Theorem 3 that

$$
\lim _{n \rightarrow \infty} P\left\{N_{m}=s \mid N(t)=n\right\}=e^{-a} \frac{a^{s}}{s !} \quad \text { for } s=0,1,2, \ldots
$$

The theorem now follows from

$$
P\left\{Y_{k, m}>\frac{a t}{m n} \mid N(t)=n\right\}=\sum_{s=0}^{k-1} P\left(N_{m}=s \mid N(t)=n\right) .
$$

We can also extend the results obtained in Theorems 3 and 4 by conditioning on $N(t) \geq n$ rather than $N(t)=n$ to get the following theorem.

THEOREM 7. Let $m=m(n)$ be a sequence of positive integers such that $m \rightarrow \infty$ and $m / n \rightarrow 0$ as $n \rightarrow \infty$. Then, for $k \geq 1,-\infty<x<\infty$ and $a>0$,

and

$$
\lim _{n \rightarrow \infty} P\left\{Y_{k, m}>\frac{a t}{m n} \mid N(t) \geq n\right\}=\sum_{s=0}^{k-1} e^{-a} \frac{a^{s}}{s !}
$$

$$
\lim _{n \rightarrow \infty} P\left\{Y_{m-k+1, m} \leq T_{n}(x) t \mid N(t) \geq n\right\}=\sum_{s=0}^{k-1} G(x) \frac{e^{-x s}}{s !} .
$$

The theorem can be proved from Theorems 3, 4 and the following lemma. 
Lemma 3. If $0<p \leq 1$, and $A=A(n), n=1,2, \ldots$, is a sequence of events such that $\lim _{n \rightarrow \infty} P(A \mid N(t)=n)=p$, then $\lim _{n \rightarrow \infty} P(A \mid$ $N(t) \geq n)=p$.

Proof. We first observe that

$$
P(A \mid N(t) \geq n)=\frac{1}{P\{N(t) \geq n\}} \sum_{k=n}^{\infty} P(A \mid N(t)=k) e^{-\lambda t} \frac{(\lambda t)^{k}}{k !} .
$$

From the proof of Theorem 2 we know that

$$
P\{N(t) \geq n\} \sim P\{N(t)=n\} \quad \text { as } n \rightarrow \infty .
$$

Moreover, it follows that as $n \rightarrow \infty$,

$$
\sum_{k=n}^{\infty} P(A \mid N(t)=k) e^{-\lambda t} \frac{(\lambda t)^{k}}{k !} \sim P(A \mid N(t)=n) P(N(t)=n) .
$$

The lemma now follows from the above.

4. The number of interarrival times satisfying certain inequalities. In this section, we consider the problem of investigating the asymptotic distribution of the number of interarrival times $Y_{j}$ lying in a given interval. Throughout this section, $m=m(n)$ denotes a sequence of positive integers such that $m \rightarrow \infty$ and $m / n \rightarrow 0$ as $n \rightarrow \infty$. Also, for $0 \leq a<b \leq 1$, let $N_{m}(a, b)$ denote the number of $Y_{j}, 1 \leq j \leq m$, that satisfy $a t<Y_{j} \leq b t$, under the condition $N(t)=n$. We now give the asymptotic distribution of the number of "large" $Y_{j}$.

TheORem 8. For $a<b, N_{m}\left(T_{n}(a), T_{n}(b)\right)$ has an asymptotic Poisson distribution with parameter $f(a, b)=e^{-a}-e^{-b}$. That is, for $k=0,1,2, \ldots$,

$$
\lim _{n \rightarrow \infty} P\left\{N_{m}\left(T_{n}(a), T_{n}(b)\right)=k \mid N(t)=n\right\}=e^{-f(a, b)} \frac{f^{k}(a, b)}{k !} .
$$

Proof. For $j=1, \ldots, n$ define the events $A_{j}^{(n)}$ by

$$
A_{j}^{(n)}=\left\{T_{n}(a) t<Y_{j} \leq T_{n}(b) t\right\} .
$$

Under $N(t)=n$, it is clear that $A_{j}^{(n)}, 1 \leq j \leq n$, are exchangeable events. Furthermore,

$$
P\left(A_{1}^{(n)} \mid N(t)=n\right)=\left(1-T_{n}(a)\right)^{n}-\left(1-T_{n}(b)\right)^{n}
$$

and

$$
\begin{aligned}
P\left(A_{1}^{(n)}\right. & \left.\cap A_{2}^{(n)} \mid N(t)=n\right) \\
& =\left(1-2 T_{n}(a)\right)^{n}-2\left(1-\frac{2 \log m+a+b}{n}\right)^{n}+\left(1-2 T_{n}(b)\right)^{n} .
\end{aligned}
$$

Then it is easy to show that 
$\lim _{n \rightarrow \infty} m P_{n}\left(A_{1}^{(n)}\right)=f(a, b) \quad$ and $\quad \lim _{n \rightarrow \infty} m^{2} P_{n}\left(A_{1}^{(n)} \cap A_{2}^{(n)}\right)=f^{2}(a, b)$.

The theorem now follows from Theorem 3 .

A similar argument gives the asymptotic distribution of the number of "small" $Y_{j}$.

TheOREM 9. For $0<a<b, N_{m}\left(\frac{a}{m n}, \frac{b}{m n}\right)$ has an asymptotic Poisson distribution with parameter $b-a$. That is,

$$
\lim _{n \rightarrow \infty} P\left\{N_{m}\left(\frac{a}{m n}, \frac{b}{m n}\right)=k \mid N(t)=n\right\}=e^{-(b-a)} \frac{(b-a)^{k}}{k !}
$$

for $k=0,1,2, \ldots$

We now extend the results of Theorems 8 and 9 to the case where we condition on $N(t) \geq n$ rather than on $N(t)=n$. Using Lemma 3 and the above theorems one can see that the following theorem holds.

THEOREM 10. (i) If $a<b$, then

$$
\lim _{n \rightarrow \infty} P\left\{N_{m}\left(T_{n}(a), T_{n}(b)\right)=k \mid N(t) \geq n\right\}=e^{-f(a, b)} \frac{f^{k}(a, b)}{k !}
$$

for $k=0,1,2, \ldots$

(ii) For $a<b$ and $k=0,1,2, \ldots$,

$$
\lim _{n \rightarrow \infty} P\left\{N_{m}\left(\frac{a}{m n}, \frac{b}{m n}\right)=k \mid N(t) \geq n\right\}=e^{-(b-a)} \frac{(b-a)^{k}}{k !} .
$$

\section{References}

[1] D. A. Darling, On a class of problems related to the random division of an interval, Ann. Math. Statist. 24 (1965), 239-253.

[2] J. Galambos, Advanced Probability Theory, Marcel Dekker, New York, 1992.

[3] - , The Asymptotic Theory of Extreme Order Statistics, Kreiger, Melbourne, Fla., 1987.

[4] D. J. Kendall, On finite and infinite sequences of exchangeable events, Studia Sci. Math. Hungar. 2 (1967), 319-327.

[5] B. Kopociński, The extreme gap in the multivariate Poisson process, Zastos. Mat. 21 (1991), 137-141.

[6] R. Pyke, Spacings, J. Roy. Statist. Soc. 27 (1965), 395-436.

[7] C. J. Ridler-Rowe, On two problems in exchangeable events, Studia Sci. Math. Hungar. 2 (1967), 415-418.

A. ABAY

DEPARTMENT OF MATHEMATICS

ROWAN COLLEGE OF NEW JERSEY

GLASSBORO, NEW JERSEY 08028

U.S.A.

E-mail: ABAY@MARS.ROWAN.EDU 